\title{
Portal Hypertension, an Overview
}

Mohammad Elmoghazy, Ahmed El Shabrawi, Nasser Mousa

\begin{abstract}
Summary
Portal hypertension $(P P)$ is the chief consequence of cirrhosis and is responsible for the majority of its complications. In seventy percentage of cases, $P P$ increases at first as a result of an increased intrahepatic resistance to portal flow attributed to structural mechanisms e.g., fibrous tissue, regenerative nodules, and micro thrombi. Yet, at least one third of the increased intrahepatic resistance is attributed to an increased intrahepatic vascular tone, which, results from endothelial dysfunction mostly mediated from reduced nitric oxide (NO) bioavailability. The onset of portal hypertension may not always be associated with specific symptoms, however, the main symptoms and complications of portal hypertension include; gastrointestinal bleeding due to the spontaneous rupture and bleeding from varices, ascites, hepatic encephalopathy and decreased levels of platelets or decreased white blood cell count. It is currently possible to diagnose liver cirrhosis and portal hypertension accurately by non-invasive methods in a reasonable proportion of patients with chronic liver disease. Recent, more complicated non-invasive diagnostic methods such as MRE, CT images and dynamic techniques on $M R I$ are emerging tools further improving this possibility.
\end{abstract}

Keywords: Portal hypertension, cirrhosis, varices, ascites, hepatic encephalopathy
Medical Journal of Viral Hepatitis

(MJVH) 2019; 4 (1) - pp. 15-21

Received: 29/6/2019

Revised: $3 / 8 / 2019$

Accepted: 5/8/2019

Published Online: 15/11/2019

(Mohammad Elmoghazy) Gastroetetrology and Hepatology dept., Damietta Cardiology and Gastroenterology center, Egypt

(Ahmed El Shabrawi, Nasser Mousa) tropical Medicine dept., Faculty of Medicine, Mansoura Univ., Egypt

* CA: Nasser Mousa

mousa_medic@yahoo.com.

\section{Definition of Portal Hypertension.}

Portal hypertension (PHT) is the hemodynamic abnormality connected with cirrhotic complications, including ascites, bleeding from gastroesophageal varices, and hepatic encephalopathy ${ }^{1}$. The normal portal venous pressure ranges between 5 and $10 \mathrm{~mm} \mathrm{Hg}$ (equivalent of 7-14 $\mathrm{cm} \mathrm{H}_{2} \mathrm{O}$ ). Portal hypertension is defined by a HVPG $>5 \mathrm{~mm} \mathrm{Hg}$, but the risk of developing GEV and the clinical complications of decompensated chronic liver disease such as; ascites, variceal bleeding and Hepatic encephalopathy (HE)) are only present when it reaches $\geq 10 \mathrm{~mm} \mathrm{\textrm {Hg } ^ { 2 }}$. Thus, $\mathrm{HVPG} \geq 10 \mathrm{~mm} \mathrm{Hg}$ is called "clinically significant portal hypertension" (CSPH). In the majority of patients, PHT is associated with increased resistance to flow within the hepatic sinusoids. The pressure gradient between the PV and the IVC represents the real perfusion pressure within the portal and hepatic circulation, which under normal conditions is a high-flow, low-resistance system, considering the high volume of portal blood flow (between 700 and $1000 \mathrm{~mL} / \mathrm{min}$ ). The different causes of an increased portal venous resistance are delineated by changes in the anatomic architecture (Fibrotic nodules, distal venous thrombosis, collagenization of the space of Disse, and loss of the normal elasticity of the sinusoidal endothelium), changes in splanchnic hemodynamics (increased splanchnic blood flow), and changes in the intrahepatic vascular resistance (vasoconstriction of the sinusoids) ${ }^{3}$.

\section{Classification and Causes of Portal Hyp- ertension}

The causes of PHT are classified into; Prehepatic, intrahepatic and posthepatic. Prehepatic 
causes of PHT including portal and splenic vein thrombosis. Posthepatic causes include those affecting the hepatic veins and venous drainage to the heart, including Budd-Chiari syndrome (BCS), veno-occlusive disease and chronic right-sided heart failure. However, intrahepatic causes comprise more than $95 \%$ of etiology of PHT and cirrhosis is the main cause. Furthermore, intrahepatic causes of PHT can be subdivided into; presinusoidal, sinu-soidal, and postsinusoidal disease, tab. (1) ${ }^{4}$.

Table (1) Causes of non-cirrhotic portal hypertension ${ }^{1}$.

\begin{tabular}{|l|l|l|}
\hline \multicolumn{1}{|c|}{ Ptrhepatic Presinusoidal PHT } & \multicolumn{1}{|c|}{ Intrahepatic PHT } & \multicolumn{1}{|c|}{$\begin{array}{c}\text { Extrahepatic Postsinusoidal } \\
\text { PHT }\end{array}$} \\
\hline - Portal vein thrombosis & * Presinusoidal & - Right heart failure \\
- Superior mesenteric vein thro-mbosis & - Hepatic schistosomiasis & - Constrictive pericarditis \\
- Splenic vein thrombosis & - Congenital hepatic fibrosis & - Suprahepatic IVC thrombosis \\
- Congestive splenomegaly (Banti's syn- & * Sinusoidal PHT & - Pulmonary hypertension \\
drome) & - Cirthosis (various causes) & - Tricuspid valve regurgitation \\
& - Noncirrhotic alcoholic liver disease & - Veno-occlusive disease \\
& - Nodular regenerative hyperplasia & - Budd-Chiari syndrome (BCS) \\
& * Postsinusoidal & \\
& - Sinusoidal obstructive syndrome & \\
\hline
\end{tabular}

\section{Stages of Cirrhosis and Portal Hypertension}

In chronic liver disease, fibrosis progresses leading to cirrhosis and continues to worsen as long as the aetiologic factor is present, fig. (1). The two main stages are the compensated and decompensated stages. The latter is characterized by the presence of clinically overt complications such as; ascites, variceal hemorrhage, or HE. The compensated stage is the longest stage, and it is asymptomatic. There are at least two main sub-stages of compensated cirrhosis with different prognostic and predominant pathophysiological mechanisms: patients with mild $\mathrm{PH}$ and those with clinically significant portal hypertension (CSPH). Patients in the latter stage are at risk of developing decompensation, particularly those who have GEV. The decompensated stage is much shorter and can rapidly progress to a stage of further decompensation in which hepatorenal syndrome (HRS) and liver failure (encephalopathy, refractory ascites and jaundice) develop leading to a high mortality ${ }^{5}$.

\begin{tabular}{|c|c|c|}
\hline $\begin{array}{l}\text { Compenstited } \\
\text { dintosis }\end{array}$ & $\begin{array}{l}\text { Decompensated } \\
\text { cinhosis }\end{array}$ & $\begin{array}{l}\text { Late(furthes) } \\
\text { decompensation }\end{array}$ \\
\hline Mild PH & Variceal & Recurrent variceal \\
\hline CSPH & & \\
\hline $\begin{array}{r}\begin{array}{r}\text { No varices } \\
\text { Varices }\end{array}\end{array}$ & $\begin{array}{l}\text { Ascites } \\
\text { HE }\end{array}$ & $\begin{array}{l}\text { Refractory ascites, } \\
\text { hyponatreemia, HRS } \\
\text { Recurrent HE } \\
\text { Jaundice }\end{array}$ \\
\hline
\end{tabular}

Figure (1) Stages and sub-stages of cirrhosis

\section{Pathogenesis of Portal Hypertension}

In cirrhosis, portal pressure (PP) increases initially as a consequence of an increased intrahepatic resistance to portal flow attributed to structural mechanisms (e.g., fibrous tissue, regenerative nodules) and an increased intrahepatic vascular tone (functional component related to endothelial dysfunction). One of the initial consequences of $\mathrm{PH}$ is the formation of portosystemic collaterals. Concomitant or even preceding development of collaterals, splanchnic vasodilatation occurs, leading to increased flow into the gut and into the portal venous system. Vasodilatation leads to activation of neurohumoral and vasoconstrictive systems, sodium and water retention, increased blood volume, and increased cardiac output; that is, a hyperdynamic circulatory state that further increases portal venous inflow and PP. Additionally, norepinephrine, angiotensin-2, and antidiuretic hormone (activated neurohumoral and vasoconstrictive systems) further contribute to intrahepatic vasoconstriction ${ }^{5,6}$. Gastric varices caused by splenic vein thrombosis (SVT) tend to arise from the short gastric veins running from the hilum of the spleen to the greater curvature aspect of the stomach rather than through splenorenal or gastrorenal shunts common with portal hypertensive fundal varices. IVC: inferior vena cava; LGV: left gastric vein; LRV: left renal vein; PV: portal vein; SGV: short gastric vein; SMV: superior mesenteric vein ${ }^{7}$. 


\section{Complications of Portal Hypertension Varices}

Cirrhotic patients often develop esophageal or gastric varices (GVs) caused by the portosystemic shunting that accompaniesPHT ${ }^{8}$. GVs usually form in the submucosal layer at the cardia or the fundus of the stomach because the posterior wall in this region of the stomach approaches the portosystemic collateral circulation. GVs receive most of their blood supply from the left, posterior, and short gastric veins and drain mainly through a gastrorenal shunt, Fig (2).

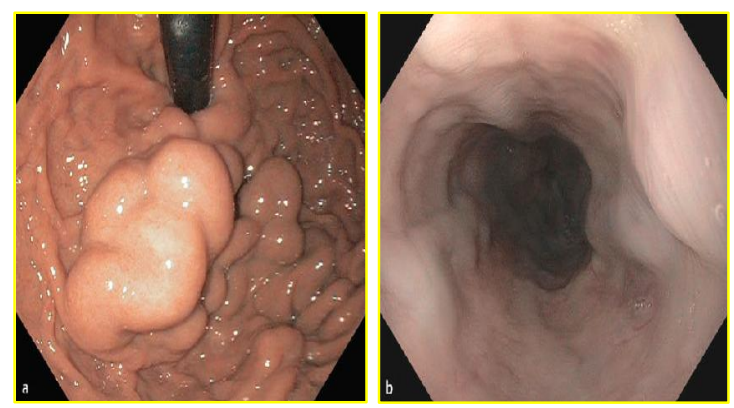

Figure (2) Esophageal and gastric varices as major complications of portal hypertension? ${ }^{9}$.

These GVs and resultant shunts predispose patients to a higher risk of experiencing a massive variceal bleeding 9 . Approximately $35 \%$ of patients with compensated cirrhosis and $80 \%$ of those with decompensated cirrhosis have varices at the time of diagnosis ${ }^{9}$. Active bleeding at the time of endoscopy is one of the hallmarks for poor prognosis in patients with varices, specially when accompanied by bacterial infection ${ }^{8}$. Mortality rates from variceal bleeding have declined in recent years but still remain high ${ }^{\mathbf{1 0}}$. PHT may also lead to portal hypertensive gastropathy (PHG), a serious condition that can cause acute or even massive blood loss. Endoscopic investigation shows abnormalities in the gastric mucosa often described as a mosaic-like patternthat resembles snake skin. The incidence of $\mathrm{PHG}$, ranging from $2 \%$ to $12 \%$, classifies this complication as a less common cause of upper gastrointestinal (GI) bleeding ${ }^{11}$. Rectal varices, frequently confused with hemorrhoids, occur in about $4 \%$ of patients with PHT. Since rectal varices seldom bleed, they are of less clinical importance than either esophageal varices or $\mathrm{GVs}^{12}$.

\section{Ascites}

Ascites develops as a consequence of PHT when fluids accumulate in the peritoneal cavity. It is a major complication of cirrhosis seen in approximately $60 \%$ of compensated patients ${ }^{12}$. Arterial splanchnic vasodilatation leads to arterial hypotension with activation of both sympathetic nervous and renin-angiotensin-aldosterone system (RAAS). In cirrhotic patients, as a consequent to the body's failure to excrete sodium into urine sufficiently, excessive sodium accumulation ultimately results in ascites development and ede$\mathrm{ma}^{13}$. Cirrhosis is the main cause of ascites (nearly $75 \%$ ). The residual $25 \%$ of ascites cases are caused by malignancy $(10 \%)$, cardiac failure $(3 \%)$, pancreatitis $(1 \%)$, tuberculosis $(2 \%)$, or other factors ${ }^{13}$. Pathogenic alterations responsible for ascites in patients with cirrhosis include two separate mechanisms ${ }^{14}$; increased portal flow resistance at the sinusoids level creating sinusoidal PHT and an associated pressure backwash into the splanchnic capillaries causes the first mechanism. Excess fluid preferentially accumulates in the peritoneal cavity and blood flow increases to the splanchnic area, leading to further increases in portal pressure. The second mechanism precedes ascites formation and relates to sustained renal sodium retention ${ }^{14}$.

\section{Hepatic Hydrothorax}

Hepatic hydrothorax $(\mathrm{HH})$ is a term to describe clinically significant pleural effusion greater than $500 \mathrm{~mL}$ in cirrhotic patients in absence of cardiac or pulmonary comorbidities ${ }^{15}$. HH usually affects the right side in the majority of cases (87\%); less commonly, it occurs in the left side or even bilateral. Of note, even small amounts of pleural effusion can cause significant respiratory embarrassment with negative impact on patient's quality of life ${ }^{16}$.

\section{Hepatorenal Syndrome}

Hepatorenal syndrome (HRS) is defined as acute kidney injury in patient with liver failure and cirrhosis as a result of significant reduction in renal perfusion ${ }^{17}$. HRS can be classified into two separate entities (types I and II). Type 2 HRS characterized by slowly progressive renal failure with average serum creatinine of $1.5 \mathrm{mg} / \mathrm{dL}$, type I HRS is found to encompass severe and rapidly 
progressive renal failure with doubling of the initial serum creatintine levels to above $2.5 \mathrm{mg} / \mathrm{dl}$ in less than two weeks ${ }^{17}$. Gradual impairment of cardiac functions observed in cirrhotic patients could be another accepted theory ${ }^{18}$. Acute on top of chronic liver failure (ACLF) with multi system affection including impairment of renal function, is a well-known associating feature that goes hand on hand with type $1 \mathrm{H}^{19}$.

\section{Hepatopulmonary Syndrome}

Patients with cirrhosis experience at some point certain vascular pulmonary complications including portopulmonary hypertention (PoPH) and hepatopulmonary syndrome (HPS) ${ }^{20}$. PoPH is a condition characterized by elevation of mean pulmonary arterial pressure (mPAP) more than $25 \mathrm{~mm} \mathrm{Hg}$ at rest or more than $30 \mathrm{~mm} \mathrm{Hg}$ during exertion; a pulmonary vascular resistance above 240 dynes.s.cm-5; and a capillary wedge pressure below $15 \mathrm{~mm} \mathrm{Hg}^{21}$ Hepatopulmonary syndrome (HPS) is a triad of liver disease, pulmonary vascular ectasia and impaired oxygenation. HPS is defined in the literature as a widened alveolar-arterial oxygen difference (Aa gradient) in room air (>15 mm Hg or $>20 \mathrm{~mm}$ $\mathrm{Hg}$ in patients > 64 years of age) with or without hypoxemia due to intrapulmonary vasodilatation in the presence of hepatic dysfunction $^{22,23}$. Clinically, patients with HPS complain of progressive dyspnea on exertion, at rest, or both. Severe hypoxemia $\left(\mathrm{PaO}_{2}<60 \mathrm{~mm} \mathrm{Hg}\right)$ is often seen and strongly suggests HPS. A classical finding in HPS is orthodeoxia defined as a decreased arterial oxygen tension by more than $4 \mathrm{~mm} \mathrm{Hg}$ or arterial oxyhemoglobin desaturation by more than 5\% with changing position from supine to standing. It is associated with platypnea defined as dyspnea worsened by upright position ${ }^{22,24}$. Orthotopic liver transplantation (OLT) remains the only definitive treatment to HPS with minor role of other strategies such as supplemental oxygen, which gives only symptomatic relief when hypoxemia supervenes can successfully reverse the condition ${ }^{25}$.

\section{Hepatic Encephalopathy}

Hepatic encephalopathy (HE) is a neurologic and psychiatric dysfunction in a patient with chronic liver disease. Impaired ability of the liver to detoxify gut-derived neurotoxins is the main cause of this condition ${ }^{26}$. Hepatic encephalopathy (HE) is a serious clinical problem of portal hypertension and cirrhosis. It is manifested by personality changes, cognitive dysfunction, and altered level of consciousness ${ }^{27,28}$. Based on the severity, HE is classified into two groups; Overt HE (OHE) presents episodically or continuously with obvious and clinically detectable symptoms. In contrast, covert HE (CHE) combines the two lowest grades of HE (minimal HE (MHE) and HE grade 1$)^{29}$. Therefore, using the new classification, OHE starts with grade 2 or with evidence of asterixis and disorientation. MHE is characterized by subtle cognitive and psychomotor deficits in the absence of recognizable clinical symptoms and signs of HE and is documented by neuro-psychometric (NP) tests and neurophysiological tests, but, HE grade 1 is defined by the presence of mild clinical alterations like euphoria, anxiety or a shortened attention span. Although the consequences are serious, mostly CHE is often unnoticed or even neglected in routine clinical practice due to only very mild symptoms associated with grade 1 , or not performed diagnostics in case of $\mathrm{MHE}^{30,31}$.

\section{Cirrhotic Cardiomyopathy}

The cirrhotic cardiomyopathy was present in about 50\% In patients with advanced cirrhosis. It is defined as a chronic cardiac dysfunction characterized by impaired contractile response and/or altered diastolic relaxation in the absence of other cardiac causes. The pathophysiology of this condition is apparently related to $\mathrm{PH}$ and hyperdynamic state in cirrhosis ${ }^{32}$. In cirrhotic patients many factors contribute to systolic and diastolic dysfunction. These factors included plasma volume expansion leading to a relative central volume decrease ${ }^{33}$, decreasing the arterial vessel wall thickness and tone leading to reduced arterial compliance $\mathrm{e}^{34,35}$ and also, autonomic dysfunction $^{36}$. Symptoms associated with cirrhotic cardiomyopathy include dyspnea with exertion, impaired exercise capacity, paroxysmal nocturnal dyspnea, peripheral edema, and orthopnea. Lessfrequent presentations include long QT on electrocardiography, arrhythmia, and sudden death ${ }^{37}$. 


\section{Diagnosis of Portal Hypertemsion}

The range of HVPG measurements can provide clinically useful information in terms of the prognosis related to portal hypertension and its complications, fig. $(3)^{38}$.

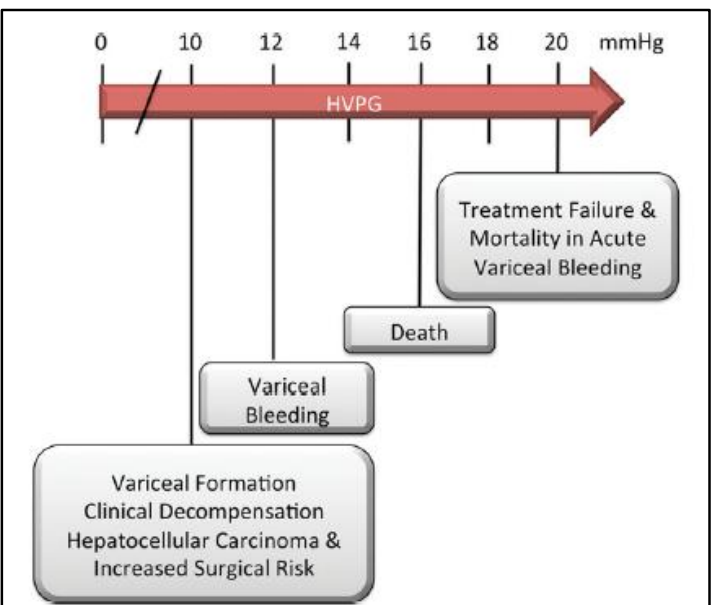

Figure (3) Diagram demonstrated the risks associated with increasing $\mathrm{HVPG}^{38}$

\section{Indirect Portal Pressure Measurement}

Measurement of HVPG remains the gold standard method to assess portal pressure (PP) through catheterization of the hepatic vein using a balloon catheter $^{39}$. Normal values of HVPG lies between 3 and $5 \mathrm{~mm} \mathrm{Hg}$. Patients with HVPG values above $5 \mathrm{mmHg}$ but less than $10 \mathrm{~mm} \mathrm{Hg}$ are defined to have mild $\mathrm{PH}$ while those with values above 10 $\mathrm{mmHg}$ are defined to have clinically significant portal hypertension (CSPH). Patients have CSPH with HVPG $>12 \mathrm{~mm} \mathrm{Hg}$ are at increased risk of variceal bleeding. Reduction of the HVPG to levels of $12 \mathrm{~mm} \mathrm{Hg}$ or below is found to have protective effect in terms of variceal hemorrhage $(\mathrm{VH})^{40}$.

\section{Noninvasive Imaging Diagnosis}

Ultrasound is the first-line imaging examination to be performed in patients with suspected cirrhosis and/or portal hypertension. Ultrasound is safe, can be repeated easily, is not expensive and is highly sensitive in detecting thrombosis in the portal vein and hepatic veins, so allowing a correct differential diagnosis of new cases of portal hypertension ${ }^{41}$. As for the limitations, inter-observer variability is considered a major drawback, but appropriate training and knowledge markedly reduce it. Intestinal gas and obesity limit the exploration. Presence of portosystemic collaterals provides the most reliable clue to diagnose portal hypertension ${ }^{42}$. Measuring liver stiffness using magnetic resonance elastography (MRE) can predict severe PHT in asymptomatic or compensated cirrhosis ${ }^{43-45}$. Evaluation of hepatic and splenic stiffness provides noninvasive modality for screening for PHT. Normal values of hepatic stiffness lies below $2.9 \mathrm{kPa}$. CSPH can be diagnosed with values $>20-25$ $\mathrm{kPa}$, with a diagnostic accuracy over $90 \%{ }^{35,36}$. However, limitations of MRE include its high cost and limited availability, which currently prevent its use as a routine diagnostic method. So that, it is currently possible to diagnose liver cirrhosis and portal hypertension accurately by non-invasive methods in a reasonable proportion of patients with chronic liver disease. However, all methods have pros and cons. New, more sophisticated non-invasive diagnostic methods such as MRE, new software analysis of images obtained with the existing technology such as the analysis of the nodularity of liver surface on ultrasound and CT images and dynamic techniques on MRI are emerging tools further improving this possibility.

\section{References}

1- Molina E, Reddy K. Noncirrhotic portal hypertension. Clin Liver Dis. 2001; 5 (3): 769 787.

2-Ripoll C, Groszmann R, Garcia-Tsao G, et al. Hepatic venous pressure gradient predicts clinical decompensation in patients with compensated cirrhosis. Gastroenterology. 2007; 133: 481- 488.

3-Pomier-Layrargues G, Huet P-M. Measurement of hepatic venous pressure gradient: Methods, interpretation, and pitfalls. In: Sanyal A, Shah V, (eds.) Portal Hypertension: Pathobiology, Evaluation, and Treatment. Totowa, NJ, Humana Press. 2005, pp 129-144.

4- Lautt W, Greenway C. Conceptual review of the hepatic vascular bed. Hepatology. 1987; 7 (5): 952-963.

5-Garcia-Tsao G, Abraldes J, Berzigotti A, Bosch J. Portal hypertensive bleeding in cirrhosis: Risk stratification, diagnosis, and management: 2016 practice guidance by the American Association for the study of liver diseases. Hepatology. 2017; 65 (1): 310-335 
6- Bosch J, Groszmann R, Shah V. Evolution in the understanding of the pathophysiological basis of portal hypertension: How changes in paradigm are leading to successful new treatments. J. Hepatol. 2015; 62 (1): 121-130.

7-Ryan B, Stockbrugger R, Ryan J. A pathophysiologic, gastroenterologic, and radiologic approach to the management of gastric varices. Gastroenterology. 2004; 126 (4): 1175-1189.

8-Garcia-Tsao G, Bosch J. Management of varices and variceal hemorrhage in cirrhosis. N Engl J. Med. 2010; 362 (9): 823-832.

9-El-Serag H, Everhart J. Improved survival after variceal hemorrhage over an11-year period in the department of veterans affairs. Am J. Gastroenterol. 2000; 95 (12): 35663573.

10- Cardenas A, Gines P. Management of patients with cirrhosis awaiting liver transplantation. Gut. 2011; 60 (3): 412-421.

11- Cubillas R, Rockey D. Portal hypertensive gastropathy: A review. Liver Int. 2010; 30 (8): 1094-1102.

12- Wiechowska-Kozlowska A, Bialek A, Milkiewicz P. Prevalence of "deep" rectalvarices in patients with cirrhosis: An EUSbased study. Liver Int. 2009; 29 (8): 12021205.

13- Pere Ginès $P$, Angeli $P$, Lenz $K$, , Moller $S$, Moore K, Moreau R. Clinical practice guidelines: EASL clinicalpractice guidelines on the management of ascites, spontaneous bacterial peritonitis, and hepatorenal syndrome in cirrhosis. J Hepatol. 2010; 53 (3): 397-417.

14- Salerno F, Guevara M, Bernardi M, Moreau R, Wong F, Angeli P, et al. Refractory ascites: Pathogenesis, definition and therapy of a severe complication in patients with cirrhosis. Liver Int. 2010; 30 (7): 937-947.

15- Hemprich U, Papadakos $P$, Lachmann B. Respiratory failure and hypoxemia inthe cirrhotic patient including hepatopulmonary syndrome. Curr Opin Anaesthesiol. 2010; 23 (2): 133-138.

16- Roussos A, Philippou N, Mantzaris G, Gourgouliannis K. Hepatic hydrothorax: Pathophysiology diagnosis and management. J. Gastroenterol Hepatol. 2007; 22 (9): 13881393.
17- Guevara M, Arroyo V. Hepatorenal syndrome. Expert Opin Pharmacother. 2011; 12 (9): 1405-1417.

18- Ruiz-del-Arbol L, Monescillo A, Arocena C, Valer $\mathrm{P}$, Ginès $\mathrm{P}$, Moreira $\mathrm{V}$, et al. Circulatory function and Hepatorenal syndrome in cirrhosis. Hepatology. 2005; 42 (2): 439-447.

19- Wasmuth H, Kunz D, Yagmur E, TimmerStranghöner A, Vidacek D, Siewert E, et al. Patients with acute on chronic liver failure display "sepsis-like" immune paralysis. J. Hepatol. 2005; 42 (2): 195-201.

20- Kochar R, Nevah Rubin M, Fallon M. Pulmonary complications of cirrhosis. Curr Gastroenterol Rep. 2011; 13 (1): 34-39.

21- Sussman N, Kochar R, Fallon M. Pulmonary complications in cirrhosis. Curr Opin Organ Transplant. 2011; 16 (3): 281-288.

22- Zhang Z, Yang C. Progress in investigating the pathogenesis of hepatopulmonary syndrome. Hepatobiliary Pancreat Dis Int. 2010; 9 (4): 355-360.

23- Mousa N. Hepatopulmonary syndrome: An Overview. J. of Gastroenterology and Hepatology Research. 2013; 2 (4): 498-502.

24- Rodríguez-Roisin R, Krowka M. Hepatopulmonary syndrome-a liver-induced lung vascular disorder. N Engl J. Med. 2008; 358 (22): 2378-87.

25- Merkel C, Bolognesi M, Sacerdoti D, Bombonato G, Bellini B, Bighin R, et al. The hemodynamic response to medical treatment of portal hypertension as a predictor of clinical effectiveness in the primary prophylaxis of variceal bleeding in cirrhosis. Hepatology. 2000; 32 (5): 930-934.

26- Ripoll C, Banares R, Rincon D, Catalina M, Lo IO, Salcedo M, et al. Influence of hepatic venous pressure gradient on the prediction of survival of patients with cirrhosis in the MELD era. Hepatology. 2005; 42 (4): 793-801.

27- Mullen K, Prakash R. New perspectives in hepatic encephalopathy. Clin Liver Dis. 2012; 16: 1-5.

28- Romero-Gómez M, Boza F, García-Valdecasas M, García E, Aguilar-Reina J. Sub clinical hepatic encephalopathy predicts the development of overt hepatic encephalopathy. Am J. Gastroenterol. 2001; 96: 2718-2723. 
29- Vilstrup H, Amodio P, Bajaj J, Cordoba J, Ferenci P, Mullen K, et al. Hepatic encephalopathy in chronic liver disease: 2014 practice guideline by the American Association for the Study of Liver Diseases and the European Association for the Study of the Liver. Hepatology. 2014; 60: 715-735.

30- Ferenci P, Lockwood A, Mullen K, Tarter R, Weissenborn K, Blei A. Hepatic encephalopathy definition, nomenclature, diagnosis, and quantification: Final report of the working party at the $11^{\text {th }}$ World Congresses of Gastroenterology, Vienna, 1998. Hepatology. 2002; 35: 716-721.

31- Labenz C, Worns M, Schattenberg J, Huber Y, Galle P, Labenz J, et al. Epidemiology of hepatic encephalopathy in german hospitalsthe EpHE study. Z Gastroenterol. 2017; 55: 741-747.

32- Zavecz J, Bueno O, Maloney R, O’Donnell J, Roerig S, Battarbee H. Cardiac excitationcontraction coupling in the portal hypertensive rat. Am. J. of Physiology-Gastrointestinal and Liver Physiology. 2000; 279 (1): G28-G39.

33- Levy M, Wexler M. Renal sodium retention and ascites formation in $\operatorname{dogs}$ with experimental cirrhosis but without portal hypertension or increased splanchnic vascular capacity. J. of Laboratory and Clinical Medicine. 1978; 91 (3): 520-536.

34- Bolognesi M, Sacerdoti D, Piva A, et al. Carbon monoxide-mediated activation of large-conductance calcium-activated potassium channels contributes to mesenteric vasodilatation in cirrhotic rats. J. of Pharmacology and Experimental Therapeutics. 2007; 321 (1): 187-194.

35- Sarkar R, Meinberg E, Stanley J, Gordon $R$, Webb R. Nitric oxide reversibly inhibits the migration of cultured vascular smooth muscle cells. Circulation Research. 1996; 78 (2): 225-230.

36- Fernandez-Munoz D, Caramelo C, Santos JC. Systemic and splanchnic hemodynamic disturbances in conscious rats with experi- mental liver cirrhosis without ascites. A. J. of Physiology-Gastrointestinal and Liver Physiology. 1985; 12 (3): G316-G320.

37- Abelmann W, Lorell B. The challenge of cardiomyopathy. J. of the American College of Cardiology. 1989; 13 (6): 1219-1239.

38- Christopher Koh and Theo Heller. Approach to the diagnosis of portal hypertension. Clinical Liver Disease. 2012, Vol. 1 (5): 133176.

39- Groszmann R, Wongcharatrawee S. The hepatic venous pressure gradient: Anything worth doing should be done right. Hepatology. 2004; 39 (2): 280-283.

40- Abraldes J, Villanueva C, Banares R, Aracil C, Catalina M, Garci A, et al. Hepatic venous pressure gradient and prognosisin patients with acute variceal bleeding treated with pharmacologic and endoscopic therapy. J. Hepatol. 2008; 48 (2): 229-236.

41- Margini C, Berzigotti A. Portal vein thrombosis: The role of imaging in the clinical setting. Dig Liver Dis. 201749 (2): 113-120.

42- Robinson K, Middleton W, Al-Sukaiti R, Teefey S, Dahiya N.. Doppler sonography of portal hypertension. Ultrasound Q. 2009; 25 (1): 3-13.

43- Friedrich-Rust $M$, Muller $C$, Winckler A, Kriener S, Herrmann E, Holtmeier J, et al. Assessment of liver fibrosis and steatosis in PBC with FibroScan, MRI, MRspectroscopy, and serum markers. J. Clin Gastroenterol. 2010; 44(1):58-65.

44- Lemoine M, Katsahian S, Ziol M,Nahon $\mathrm{P}$, Ganne-Carrie N, Kazemi F et al. Liver stiffness measurement as a predictive tool of clinically significant portal hypertension in patients with compensated hepatitis $\mathrm{C}$ virus or alcohol-related cirrhosis. Aliment Pharmacol Ther. 2008; 28 (9): 1102-1110.

45- Vizzutti F, Arena U, Romanelli R,Rega L, Foschi M, Colagrande S, et al. Liver stiffness measurement predicts severe portal hypertension in patients with $\mathrm{HCV}$ related cirrhosis. Hepatology. 2007; 45 (5): 1290-1297. 